Supporting information

\title{
Pre-Solid Electrolyte Interphase-Covered Li Metal Anode with Improved Electro- Chemo-Mechanical Reliability in High-Energy-Density Batteries
}

\author{
Xi Chen, Mingwei Shang, Junjie Niu* \\ Department of Materials Science and Engineering, CEAS, University of Wisconsin-Milwaukee, Milwaukee, \\ WI 53211, USA \\ *Email: niu@uwm.edu
}

This file includes:

Figures S1 to S12 with legends

Tables S1 to S2 


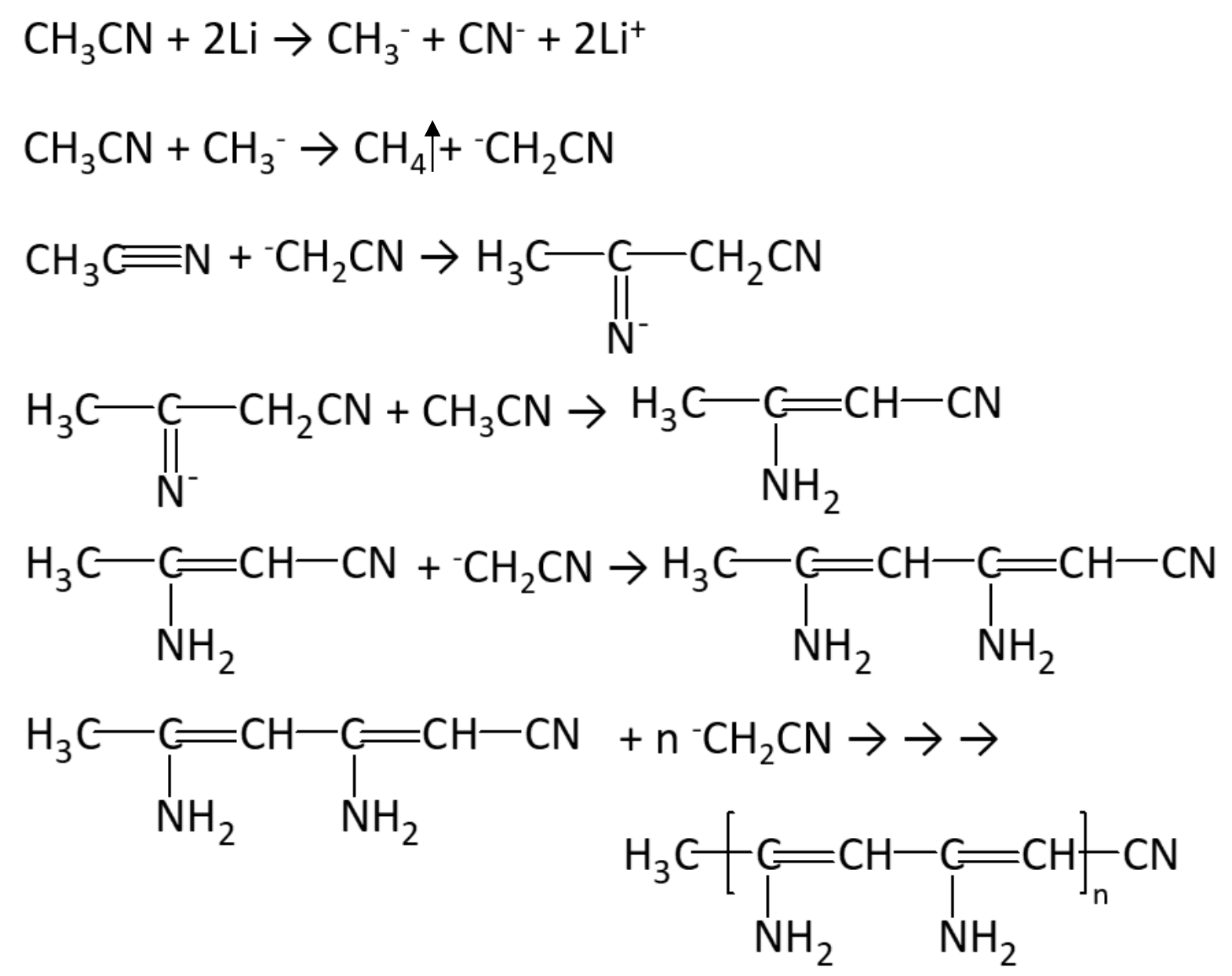




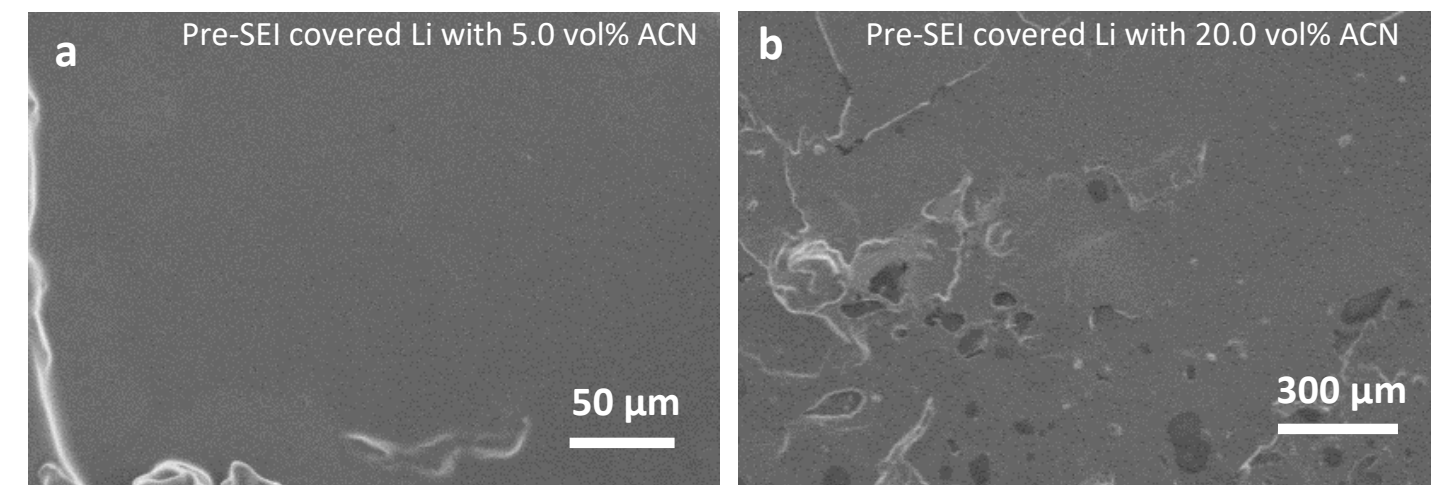

Figure S2. SEM images of the as-received pre-SEl covered Li metals using 5.0 vol\% (a) and 20.0 vol\% (b) ACN with $1.0 \mathrm{M}$ LiTFSI in HFE solution, respectively. 

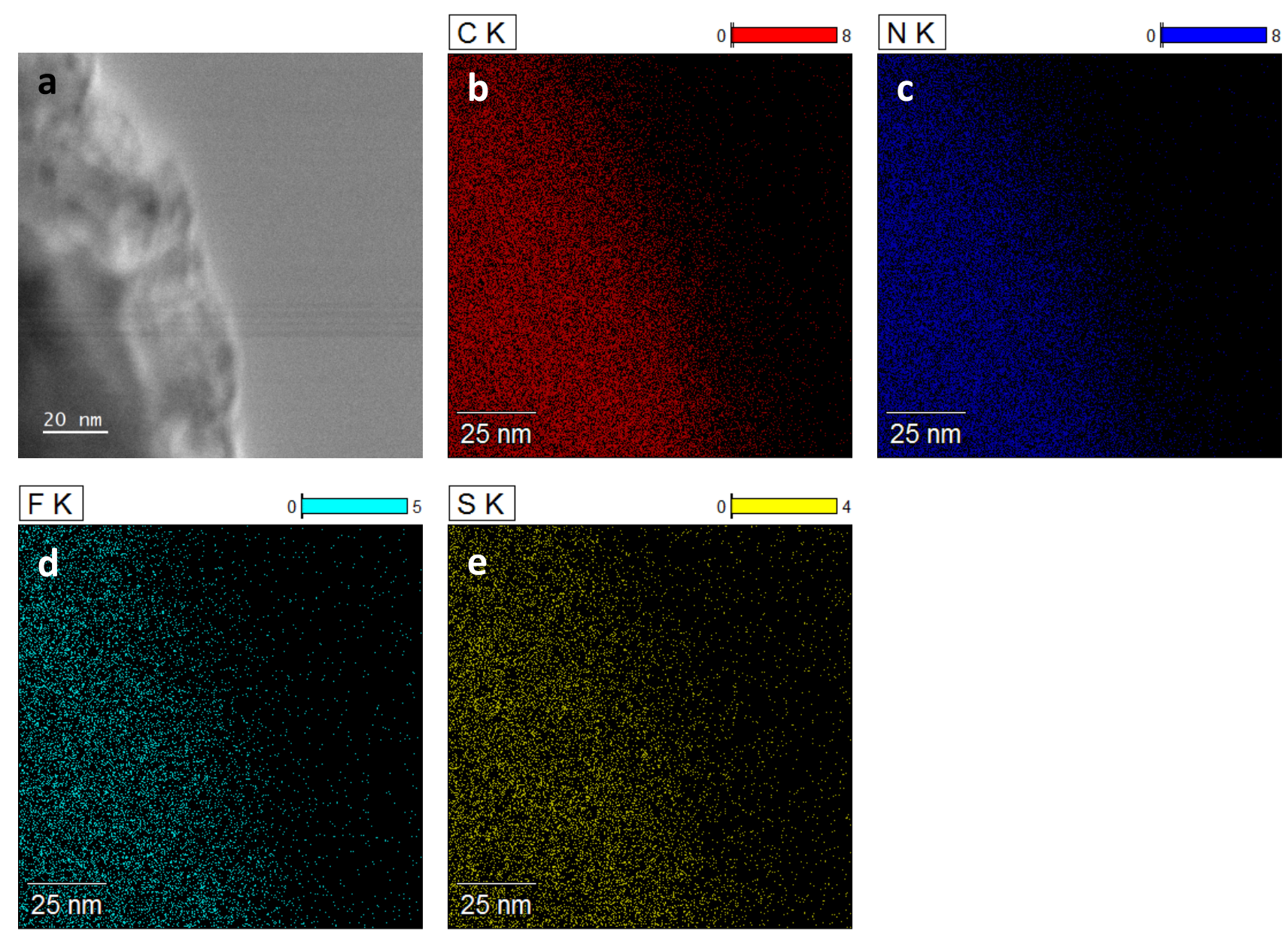

Figure S3. STEM bright-field image of the pre-SEI layer (a) and the corresponding EDS elemental mappings (b-e). 


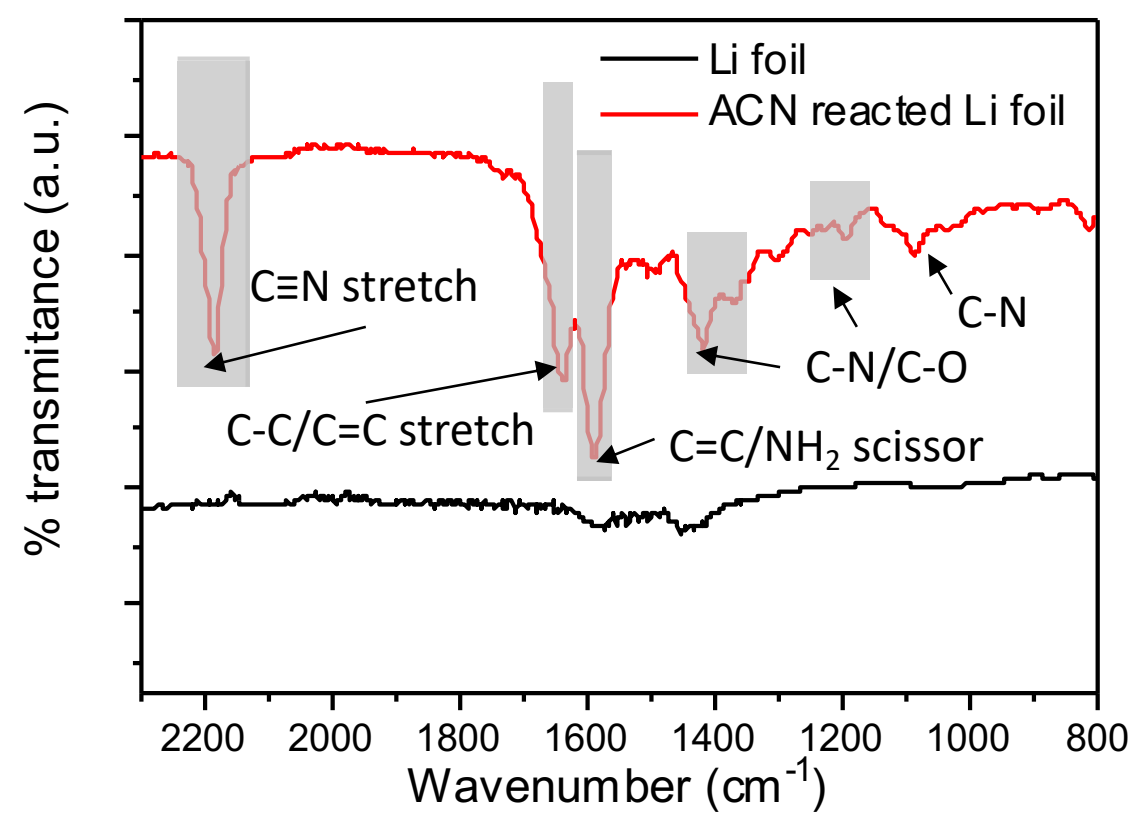

Figure S4. FTIR spectra of pure Li foil and ACN reacted Li foil. 30 ul ACN solution was dropped onto a polished Li foil with a diameter of $1.56 \mathrm{~cm}$, and then dried in Ar over 24 hours. 

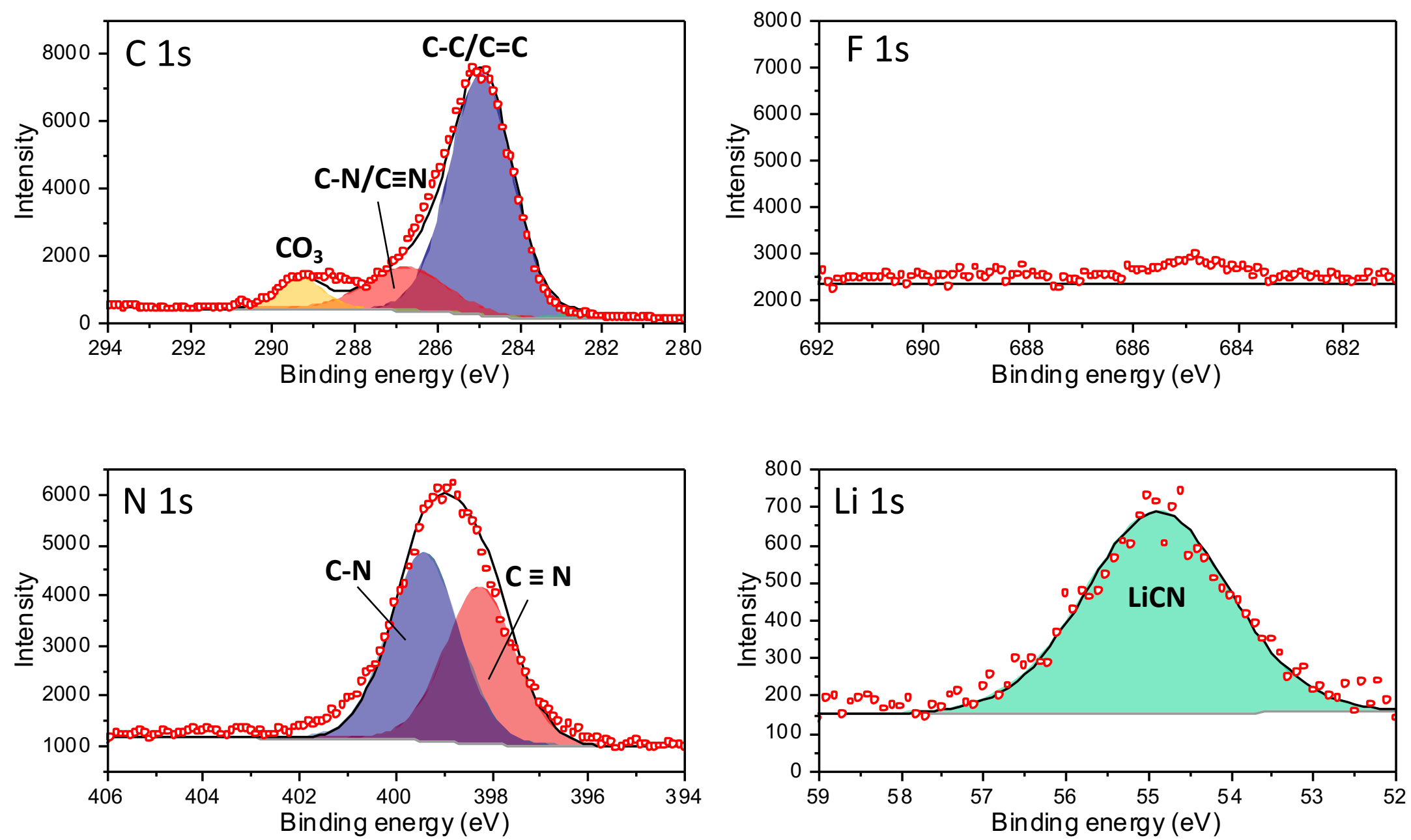

Figure S5. High-resolution XPS spectra of C1s, F1s, Li1s and N1s with pure ACN reacted Li metal surface. 

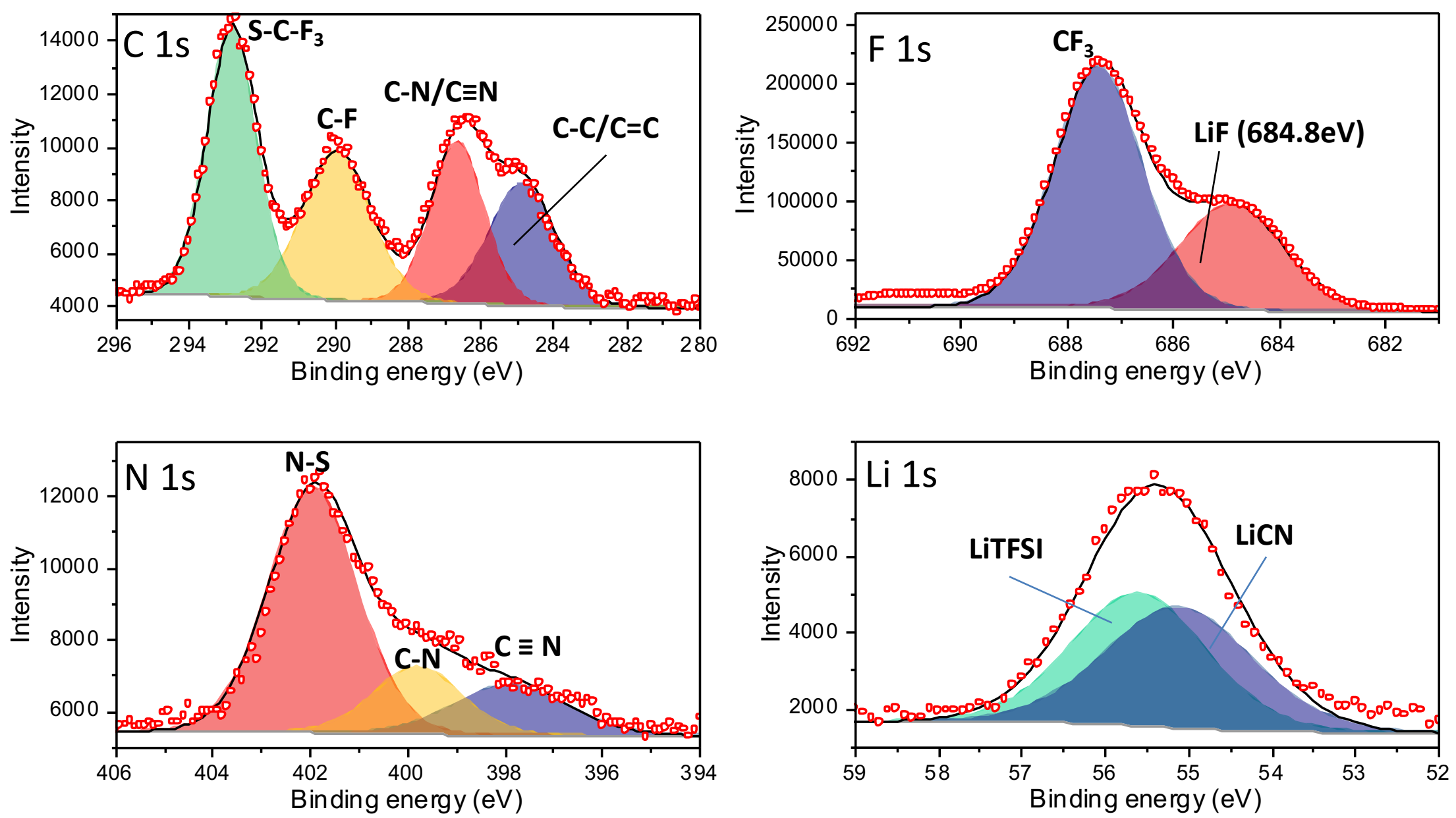

Figure S6. High-resolution XPS spectra of the Li metal surface after reacting with 1.0 M LiTFSI in ACN solution. 

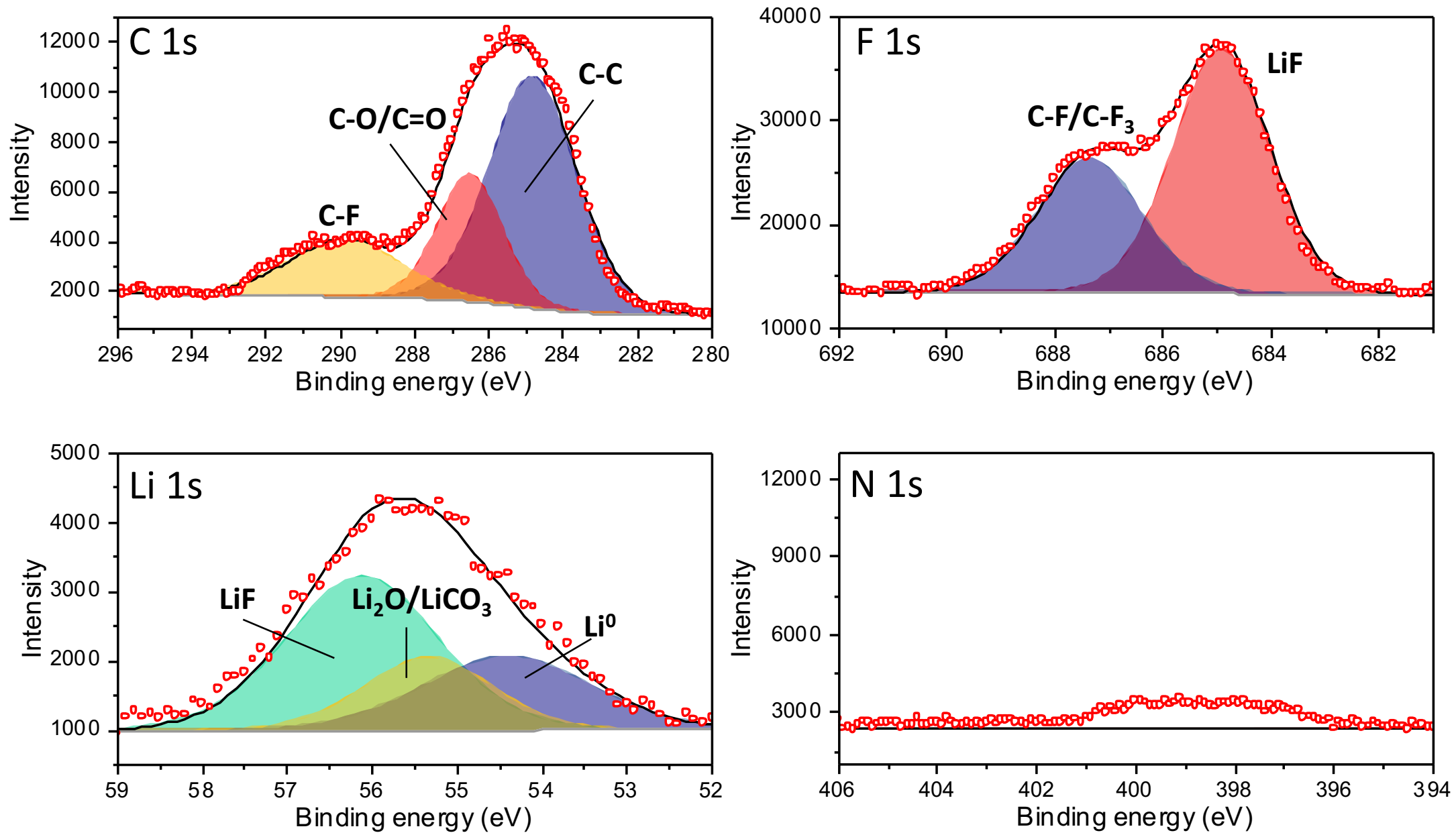

Figure S7. High-resolution XPS spectra of the Li metal surface after reacting with pure HFE solution. 

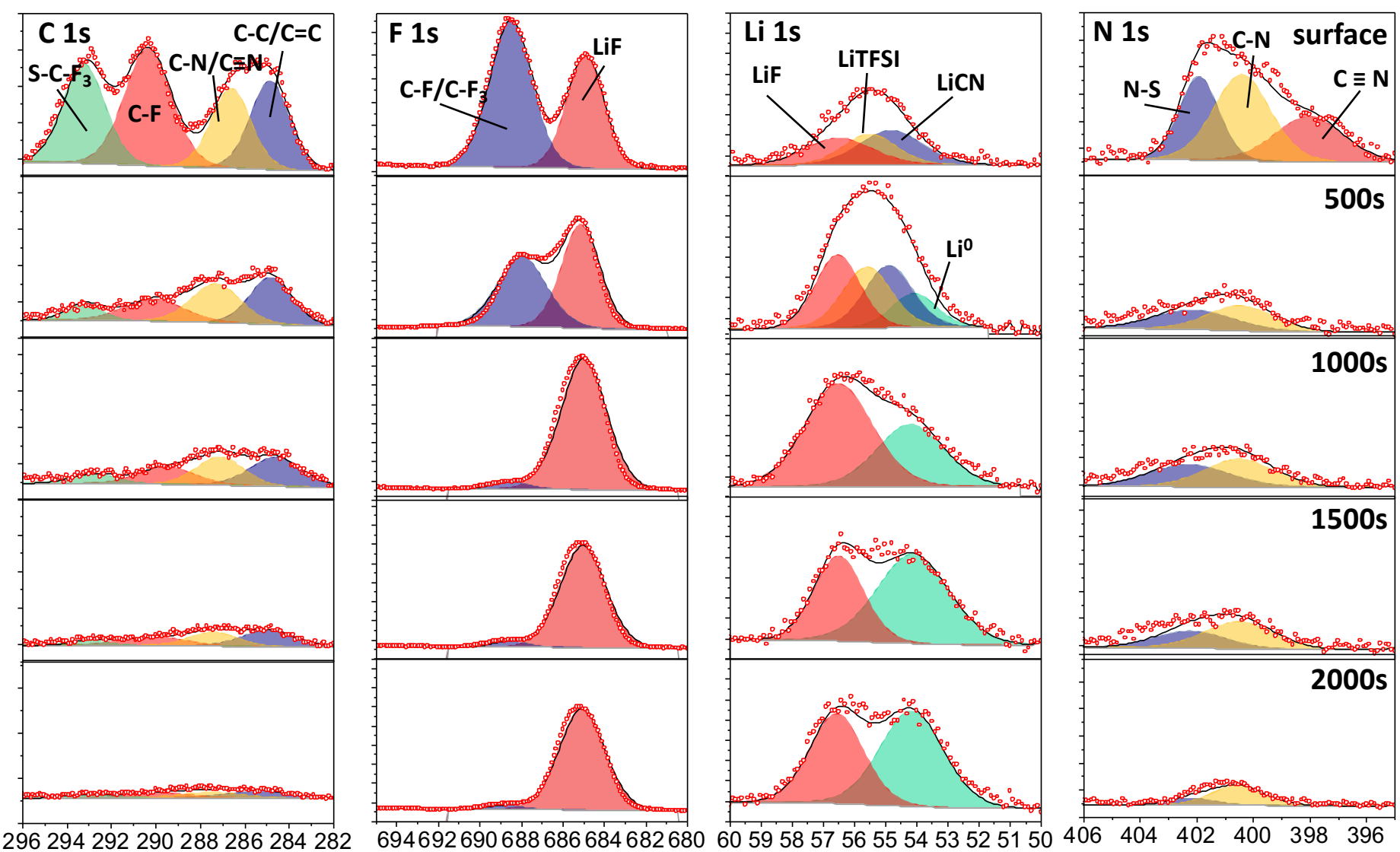

Figure S8. High-resolution XPS spectra depth profiles of the Li metal with reinforced artificial pre-SEI. 




Figure S9. High-resolution XPS spectra depth profiles of the traditional Li metal upon stripping after 3 cycles in symmetric cells. Electrolyte: $1.0 \mathrm{M} \mathrm{LiPF}_{6}$ dissolved in 3:7 weight ratio of EC and

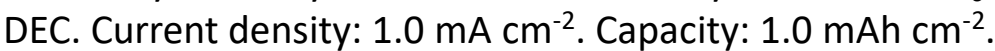






Figure S10. Rate performance of symmetric cells with pre-SEl covered Li. The precursors are 10.0 vol\% ACN, 1.0 M LiTFSI in HFE solution. The current density ranges from 0.5 to $10.0 \mathrm{~mA} \mathrm{~cm}{ }^{-2}$ and the capacity ranges from 0.5 to $10.0 \mathrm{mAh} \mathrm{cm}^{-2}$, respectively. 

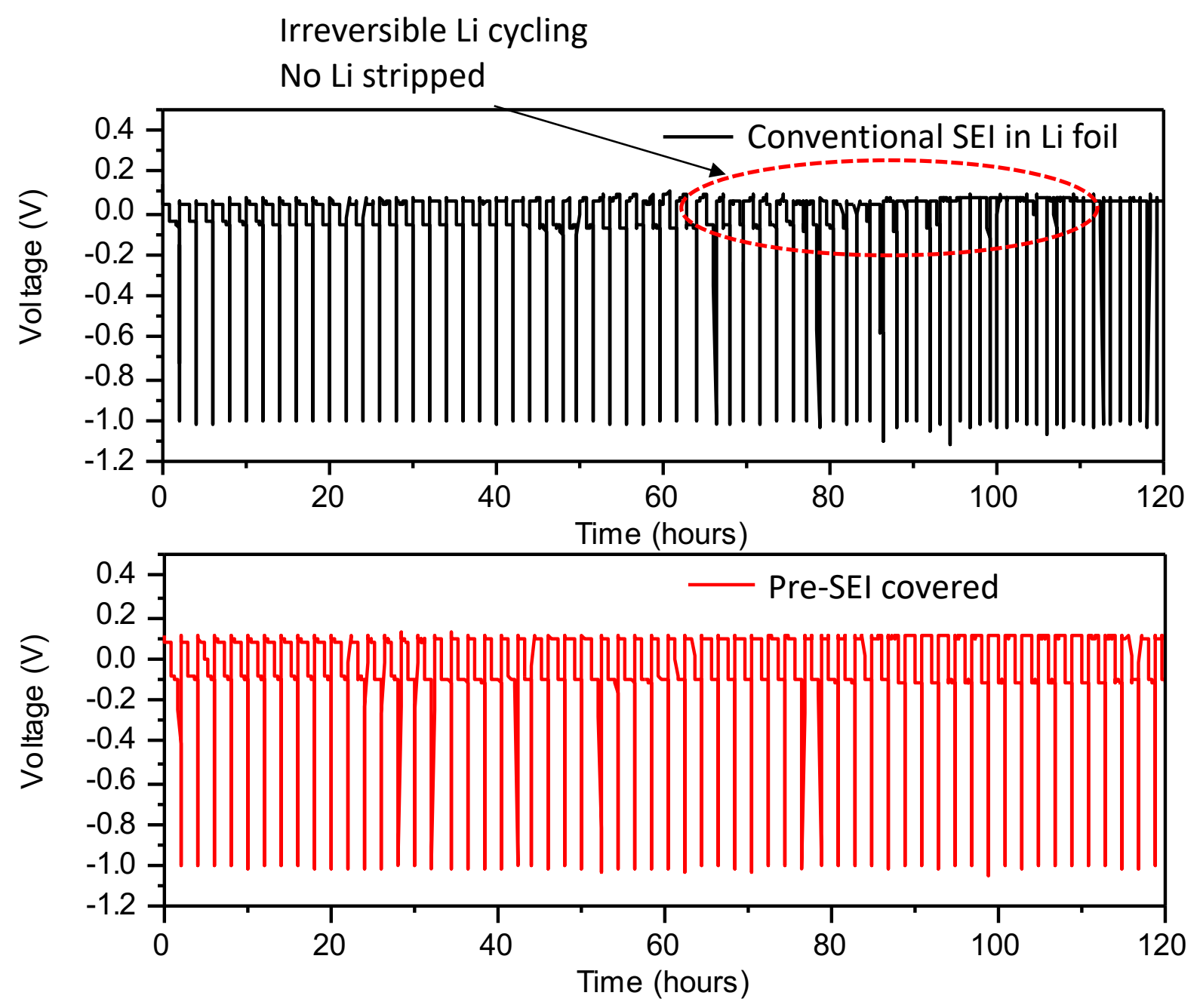

Figure S11. Voltage profiles of $\mathrm{CU}$ Li symmetric cells with and without the pre-SEI. The pre-SEI was prepared using the precursors of 10.0 vol\% ACN, 1M LiTFSI in HFE. The plating current density: $3.0 \mathrm{~mA} \mathrm{~cm}$ 2. Capacity: $3.0 \mathrm{mAh} \mathrm{cm}^{-2}$. Stripping cutoff voltage: $1.0 \mathrm{~V}$. Electrolyte: $1.0 \mathrm{M} \mathrm{LiPF}_{6}$ in EC:DEC weight ratio of 3:7. Electrolyte amount: $60 \mu \mathrm{l}$ per symmetric cell. 

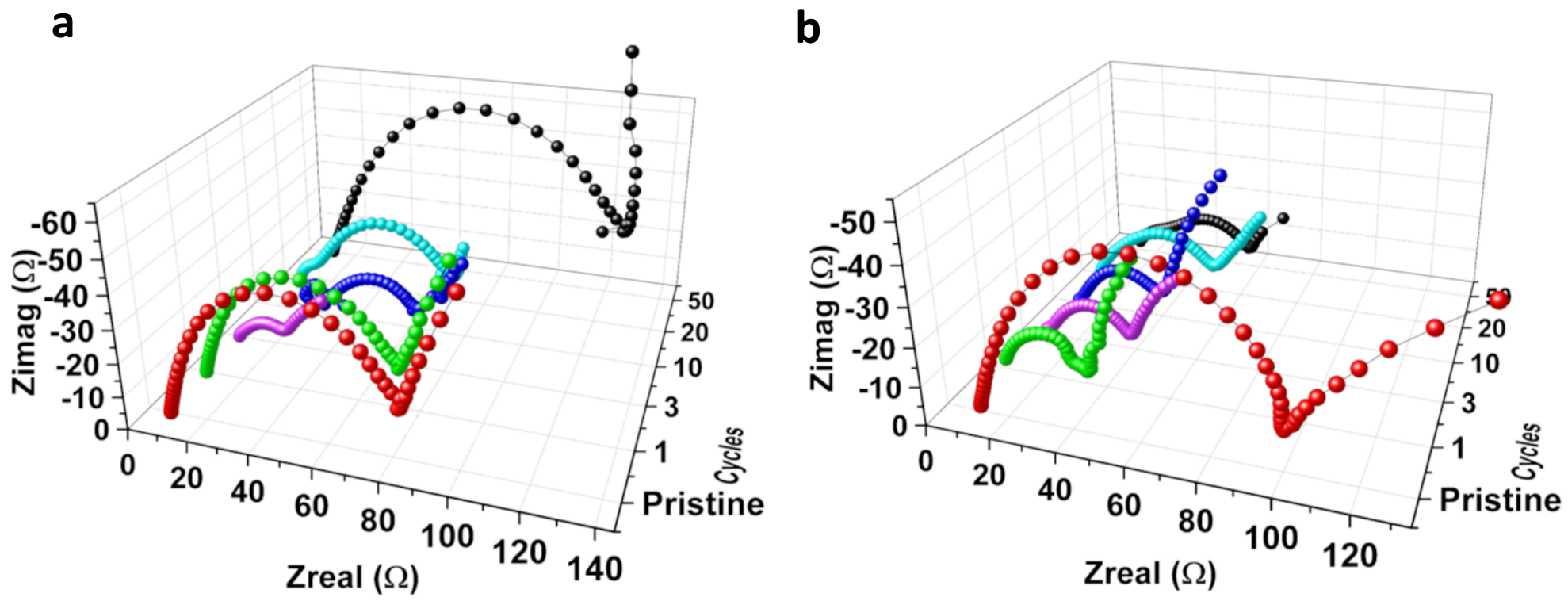

Figure S12. Nyquist plots of Li foil (a) and pre-SEl covered Li (b) vs NMC 532 full cells from EIS measurements. 


\section{Pre-SEI covered Li (30 micron) vs NCA (loading: 20.5 mg cm}

\begin{tabular}{|c|c|c|c|}
\hline Cathode areal capacity & $3.92 \mathrm{mAh} \mathrm{cm}^{-2}$ & $3.92 \mathrm{mAh} \mathrm{cm}^{-2}$ & $3.92 \mathrm{mAh} \mathrm{cm}^{-2}$ \\
\hline Anode areal capacity & $\begin{array}{l}6.2 \mathrm{mAh} \mathrm{cm}^{-2}(\sim 30 \\
\text { micron Li foil })\end{array}$ & $\begin{array}{l}6.2 \mathrm{mAh} \mathrm{cm} \mathrm{cm}^{-2}(\sim 30 \text { micron } \mathrm{Li} \\
\text { foil })\end{array}$ & $\begin{array}{l}6.2 \mathrm{mAh} \mathrm{cm}^{-2}(\sim 30 \text { micron } \mathrm{Li} \\
\text { foil })\end{array}$ \\
\hline N/P ratio & $\sim 1.5$ & $\sim 1.5$ & $\sim 1.5$ \\
\hline $\mathrm{E} / \mathrm{C}$ ratio & $1.5 \mathrm{~g}(\mathrm{Ah})^{-1}$ & $3.0 \mathrm{~g}(\mathrm{Ah})^{-1}$ & $6.0 \mathrm{~g}(\mathrm{Ah})^{-1}$ \\
\hline Discharge capacity & $\sim 185.0 \mathrm{mAh} \mathrm{g}^{-1}$ & $\sim 185.0 \mathrm{mAh} \mathrm{g}^{-1}$ & $\sim 185.0 \mathrm{mAh} \mathrm{g}^{-1}$ \\
\hline $\begin{array}{l}\text { Average discharge } \\
\text { voltage }\end{array}$ & $3.85 \mathrm{~V}$ & $3.85 \mathrm{~V}$ & $3.85 \mathrm{~V}$ \\
\hline Cathode (D:1.58 cm) & $\begin{array}{l}41.0 \mathrm{mg}+2.2 \mathrm{mg} \text { (carbon } \\
\text { and binder) }\end{array}$ & $\begin{array}{l}41.0 \mathrm{mg}+2.2 \mathrm{mg} \text { (carbon and } \\
\text { binder) }\end{array}$ & $\begin{array}{l}41.0 \mathrm{mg}+2.2 \mathrm{mg} \text { (carbon and } \\
\text { binder) }\end{array}$ \\
\hline $\mathrm{Al}$ foil (T: $25 \mu \mathrm{m})$ & $6.6 \mathrm{mg}$ & $6.6 \mathrm{mg}$ & $6.6 \mathrm{mg}$ \\
\hline Electrolyte & $\begin{array}{l}10.0 \mu \mathrm{l}, 12.0 \mathrm{mg}, 1.25 \mu \mathrm{l} \\
(\mathrm{mAh})^{-1}\end{array}$ & $\begin{array}{l}20.0 \mu 1,24.0 \mathrm{mg}, 2.5 \mu \mathrm{l} \\
(\mathrm{mAh})^{-1}\end{array}$ & $40.0 \mu \mathrm{l}, 48.0 \mathrm{mg}, 5.0 \mu \mathrm{l}(\mathrm{mAh})^{-1}$ \\
\hline Separator & $3.4 \mathrm{mg}$ & $3.4 \mathrm{mg}$ & $3.4 \mathrm{mg}$ \\
\hline Anode (D: $1.56 \mathrm{~cm}$ ) & $\begin{array}{l}5.8 \mathrm{mg}(2.7 \mathrm{mg} \text { pre-SEI } \\
\text { and side products }+3.1 \mathrm{mg} \\
\mathrm{Li})\end{array}$ & $\begin{array}{l}5.8 \mathrm{mg}(2.7 \mathrm{mg} \text { pre-SEI and } \\
\text { side products }+3.1 \mathrm{mg} \mathrm{Li})\end{array}$ & $\begin{array}{l}5.8 \mathrm{mg}(2.7 \mathrm{mg} \text { pre-SEI and side } \\
\text { products }+3.1 \mathrm{mg} \mathrm{Li})\end{array}$ \\
\hline $\begin{array}{l}\text { Estimated package foil } \\
\text { and tabs }(\sim 3 \mathrm{wt} \%)\end{array}$ & $12.1 \mathrm{mg}$ & $2.38 \mathrm{mg}$ & $3.21 \mathrm{mg}$ \\
\hline Total weight & $73.1 \mathrm{mg}$ & $81.98 \mathrm{mg}$ & $110.2 \mathrm{mg}$ \\
\hline Energy density & $404.8 \mathrm{Wh} \mathrm{kg}^{-1}$ & $360.9 \mathrm{Wh} \mathrm{kg}^{-1}$ & $268.5 \mathrm{Wh} \mathrm{kg}^{-1}$ \\
\hline
\end{tabular}

Table S1. Coin cell energy density estimation. The cathode areal capacity and average discharge voltage of the cell were collected after initial activation. The anode areal capacity was calculated based on the volume and density of Li metal. Discharge capacity was the average value of the first 50 cycles. The cases were excluded in the calculation. The electrolyte was $1.0 \mathrm{M} \mathrm{LiPF}_{6}$ dissolved in $3: 7$ weight ratio of EC and EMC with $2.0 \mathrm{wt} \%$ vinylene carbonate (VC) and $10.0 \mathrm{wt} \%$ fluoroethylene carbonate (FEC). All other cell components were considered as a standard coin cell CR2032. 


\section{Pre-SEI covered Li (30 micron) vs NMC532 (loading: $24.2 \mathrm{mg} \mathrm{cm}^{-2}$, double-side coating)}

\begin{tabular}{|c|c|c|}
\hline & $300 \mathrm{mAh}$ level pouch cell & $150 \mathrm{mAh}$ level pouch cell \\
\hline Cathode areal capacity & $3.87 \mathrm{mAh} \mathrm{cm}^{-2}$ & $3.87 \mathrm{mAh} \mathrm{cm}^{-2}$ \\
\hline Anode areal capacity & $\begin{array}{l}12.4 \mathrm{mAh} \mathrm{cm}^{-2}(\sim 30 \text { micron pre-SE } \\
\text { covered } \mathrm{Li})\end{array}$ & $\begin{array}{l}\text { I } 12.4 \mathrm{mAh} \mathrm{cm}^{-2}(\sim 30 \text { micron pre-SEI } \\
\text { covered } \mathrm{Li})\end{array}$ \\
\hline $\mathrm{N} / \mathrm{P}$ ratio & $\sim 3.0$ & $\sim 3.0$ \\
\hline $\mathrm{E} / \mathrm{C}$ ratio & $6.0 \mathrm{~g}(\mathrm{Ah})^{-1}$ & $6.0 \mathrm{~g}(\mathrm{Ah})^{-1}$ \\
\hline Discharge capacity & $\sim 160.0 \mathrm{mAh} \mathrm{g}^{-1}$ & $\sim 160.0 \mathrm{mAh} \mathrm{g}^{-1}$ \\
\hline Average discharge voltage & $3.85 \mathrm{~V}$ & $3.85 \mathrm{~V}$ \\
\hline Cathode & $\begin{array}{l}5 \times 4 \mathrm{~cm}, 4 \text { sheets, total } 80 \mathrm{~cm}^{2} \\
1.936 \mathrm{~g} \text { NMC } 532+119 \mathrm{mg} \text { (carbon } \\
\text { and binder) }\end{array}$ & $\begin{array}{l}5 \times 2 \mathrm{~cm}, 4 \text { sheets, total } 40 \mathrm{~cm}^{2} \\
968 \mathrm{mg} \text { NMC } 532+59.6 \mathrm{mg} \text { (carbon and } \\
\text { binder) }\end{array}$ \\
\hline Al foil (T: $15 \mu \mathrm{m})$ & $324 \mathrm{mg}$ & $162 \mathrm{mg}$ \\
\hline Electrolyte & $1.86 \mathrm{~g}, 6.0 \mathrm{~g}(\mathrm{Ah})^{-1}$ & $0.94 \mathrm{~g}, 6.0 \mathrm{~g}(\mathrm{Ah})^{-1}$ \\
\hline Separator & $98.5 \mathrm{~cm}^{2}, 121.5 \mathrm{mg}$ & $52.2 \mathrm{~cm}^{2}, 64.3 \mathrm{mg}$ \\
\hline $\begin{array}{l}\text { Anode }(30 \mu \mathrm{m} \text { thin } \mathrm{Li} \text { foil } \\
\text { coated on copper foil) }\end{array}$ & $\begin{array}{l}485.8 \mathrm{mg} \text { ( } \mathrm{Li} \text { foil }+\mathrm{SEI}, 5 \times 4 \mathrm{~cm}, 3 \\
\text { layers of double-side pre-SEI } \\
\text { covered Li and } 2 \text { layers of single- } \\
\text { side pre-SEI covered Li ) }\end{array}$ & $\begin{array}{l}242.9 \mathrm{mg} \text { ( } \mathrm{Li} \text { foil }+\mathrm{SEI}, 5 \times 2 \mathrm{~cm}, 3 \text { layers } \\
\text { of double-side pre-SEI covered } \mathrm{Li} \text { and } 2 \\
\text { layers of single-side pre-SEI covered } \mathrm{Li} \text { ) }\end{array}$ \\
\hline $\mathrm{Cu}$ foil (T: $9 \mu \mathrm{m})$ & $804.6 \mathrm{mg}$ & $402.3 \mathrm{mg}$ \\
\hline Package foil & $144 \mathrm{~cm}^{2}, 297.5 \mathrm{mg}$ & $108 \mathrm{~cm}^{2}, 223.1 \mathrm{mg}$ \\
\hline Tabs & $160 \mathrm{mg} \mathrm{Ni}$ tab $+60 \mathrm{mg} \mathrm{Al}$ tab & $160 \mathrm{mg} \mathrm{Ni}$ tab $+60 \mathrm{mg} \mathrm{Al}$ tab \\
\hline Total weight & $6168.4 \mathrm{mg}$ & $3282.2 \mathrm{mg}$ \\
\hline Energy density & 193.3 $\mathrm{Wh} \mathrm{kg}^{-1}$ & 181.6 $\mathrm{Wh} \mathrm{kg}^{-1}$ \\
\hline
\end{tabular}

Table S2. Pouch cell energy density estimation. The cathode areal capacity, discharge capacity and average discharge voltage of the cells were collected after initial activation. Anode areal capacity was calculated based on the volume and density of Li metal. The electrolyte was $1.0 \mathrm{M} \mathrm{LiPF}_{6}$ dissolved in 3:7 weight ratio of EC and EMC with 2.0 wt\% VC and 10.0 wt\% FEC. 